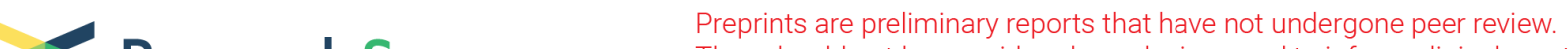

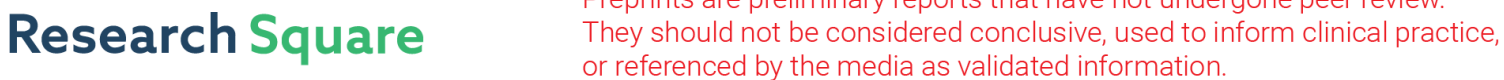

\section{Molecular Basis for RNA Polymerase-Dependent Transcription Complex Recycling By The Helicase- like Motor Protein HelD.}

\section{Timothy Newing}

University of Wollongong https://orcid.org/0000-0002-7147-6984

\section{Aaron Oakley}

University of Wollongong https://orcid.org/0000-0002-4764-014X

\section{Michael Miller}

University of Newcastle Australia https://orcid.org/0000-0001-7990-3381

\section{Catherine Dawson}

University of Newcastle Australia https://orcid.org/0000-0002-9780-4130

\section{Simon Brown}

University of Wollongong https://orcid.org/0000-0003-3997-4614

James Bouwer

University of Wollongong https://orcid.org/0000-0003-2384-070X

\section{Gokhan Tolun}

University of Wollongong

Peter Lewis ( $\sim$ Peter.Lewis@newcastle.edu.au )

University of Newcastle Australia https://orcid.org/0000-0002-1992-062X

\section{Article}

Keywords: Bacillus subtilis, RNA polymerase (RNAP), DNA replication

Posted Date: July 16th, 2020

DOI: https://doi.org/10.21203/rs.3.rs-40738/v1

License: (1) (1) This work is licensed under a Creative Commons Attribution 4.0 International License.

Read Full License

Version of Record: A version of this preprint was published at Nature Communications on December 1st, 2020. See the published version at https://doi.org/10.1038/s41467-020-20157-5. 


\section{Abstract}

In bacteria, transcription complexes stalled on DNA represent a major source of roadblocks for the DNA replication machinery that must be removed in order to prevent damaging collisions. Gram-positive bacteria contain a transcription factor HelD that is able to remove and recycle stalled complexes, but it was not known how it performed this function. Here, using cryo-electron microscopy and single-particle analysis, we have determined the structures of Bacillus subtilis RNA polymerase (RNAP) elongation and HelD complexes, enabling analysis of the extraordinary conformational changes that occur in RNAP driven by HelD interaction. HelD has a unique 2-armed structure which penetrates deep into the primary and secondary channels of RNA polymerase. One arm removes nucleic acids from the active site, and the other induces a dramatic conformational change in the primary channel leading to removal and recycling of the stalled polymerase.

\section{Main Text}

In bacteria, transcription and DNA replication occur concomitantly, making potentially damaging collisions of DNA replication forks with transcription complexes inevitable ${ }^{1-5}$. Transcription is highly sensitive to DNA damage, which causes the elongation complex (EC) to pause, and multiple redundant systems have evolved to ensure rapid removal of RNAP and/or the repair of damaged DNA ${ }^{6-10}$. This reduces the chance of replication forks colliding with stalled transcription complexes whilst also serving as an efficient system for maintaining genome integrity, especially within coding regions. However, independent of DNA damage, $\sim 15 \%$ of paused transcription complexes are inactive for a significant period of time ${ }^{11}$ and require removal through the action of factors such as HelD ${ }^{12}$. When used in a multiround transcription assay, the effect of HelD is to increase transcription activity through the recycling of non-productive complexes that have stalled on the template DNA ${ }^{12}$ indicating it is also important in recycling RNAP.

HelD is widely distributed in Gram-positive bacteria and has superficial similarity to superfamily 1 (SF1) DNA helicases such as UvrD/PcrA, and, its catalytic activity is ATP-dependent ${ }^{12}$. Low-resolution smallangle $\mathrm{X}$-ray scattering data indicate that HelD undergoes an ATP-dependent conformational change and is capable of binding to $D_{N A}{ }^{13}$, suggesting that these are important properties of HelD in transcription complex recycling. SF1 helicases UvrD/PcrA bind on the upstream side of RNAP, and are able to reversetranslocate it away from a site of DNA damage ${ }^{6,14}$. Similarly, Rad26 (eukaryotic) and RapA (prokaryotic), Swi2/Snf2 family helicases also bind on the upstream side of RNAP and reverse-translocate stalled complexes during their reactivation ${ }^{15,16}$. Although HelD has been shown to bind on the downstream side of RNAP ${ }^{12}$, it seemed reasonable to assume that HelD may facilitate transcription complex recycling in a similar manner to UvrD/PcrA, RapA and Rad26, utilising ATP-dependent translocation of stalled complexes along a DNA template. 
In this work, we show this is not the case and that HelD has a novel and remarkable structure that enables an equally remarkable mode of activity to recycle transcription complexes involving dramatic conformational change. Using cryo-electron microscopy and single-particle analysis (cryo-EM), we determined the structures of Bacillus subtilis RNA polymerase (RNAP) elongation and HelD complexes, enabling analysis of the extraordinary conformational changes that occur in RNAP driven by HelD interaction. HelD represents an unusual new class of motor protein distantly related to the SF1 helicases, containing two arms that flank the helicase-like domains. One arm anchors the rest of HelD and binds deep within the secondary channel of RNAP where it sterically clashes with nucleic acids in the active site and in doing so distorts the highly conserved bridge helix of RNAP. The other arm pushes open the primary DNA-binding channel of RNAP, causing a conformational change that releases bound DNA. Thus, HelD is a prototypical member of a widely dispersed and divergent branch of the SF1 helicase family that maintain genome integrity by removing non-productive transcription roadblocks.

\section{Structure determination of the transcription elongation complex}

Bacillus subtilis is the model representative organism of the medically and industrially important Firmicutes phylum that have genomes with a low $\mathrm{G}+\mathrm{C}$ content. Despite considerable effort, no structure of RNAP from the low $G+C$ Gram positives has been determined to date. RNAP core $\left(a_{2} \beta \beta \omega\right)$ was purified (Extended Data Fig. 1), and it's activity established using a HelD-dependent stimulation of multi-round transcription assay which gave identical results to those observed in previous studies ${ }^{12}$ (Fig. 1a). We then used cryo-EM to determine the structure of the $B$. subtilis RNAP transcription elongation complex (EC) at $3.36 \AA$, to enable an understanding of the conformational changes caused by HelD during transcription complex recycling (Fig. 1b and c, Table 1; Extended Data Fig. 2, Movie 1). RNAP in the Firmicutes is the smallest multi-subunit polymerase ${ }^{17,18}$, and given the industrial and clinical importance of this group of bacteria this complex will serve as an invaluable reference structure. 
Table 1

Cryo-EM data collection, refinement and validation statistics

RNAP Elongation Complex (EMD-

21920, PDB 6WVJ)
RNAP-HelD Complex (EMD21921, PDB 6WVK)

\section{Data Collection and Processing}

Molecular Mass (kDa)

Magnification

Voltage $(\mathrm{kV})$

Electron exposure $\left(\mathrm{e}^{-} \AA^{-2}\right)$

Defocus Range $(\mu \mathrm{m})$

Pixel size $(\AA)$

Symmetry imposed

Initial particle images (no.)

Final particle images (no.)

Relative abundance (\%)

Map resolution $(\AA ̊)$

FSC threshold

Dimensions (Length $x$ width $\times$ hight in $\AA$ )

\section{Refinement}

Initial model used

Model resolution $(\AA)$

FSC threshold

Model composition

Non-hydrogen atoms

Protein residues

Nucleic acid residues

Ligands

B Factors $\left(\AA^{2}\right)$

Protein

63.03
442.060

59,524

300

63.6

$0.6-2.8$

0.84

C1

580,468

65,356

$11.2 \%$

3.36

0.143

$156 \times 154 \times 139$
6WVK

3.38

0.5

22367

2802

37

ZN: 2, MG: 1

ZN: 2, MG: 1
28225

3631

$4 \mathrm{NJC}(\varepsilon)$

3.27

0.5 


\section{RNAP Elongation Complex (EMD- RNAP-HelD Complex (EMD- \\ 21920, PDB 6WVJ) \\ 21921, PDB 6WVK)}

Nucleic

Ligand

r.m.s deviations

Bond lengths $(\AA)$

0.005

0.692

0.005

Bond angles $\left({ }^{\circ}\right)$

111.00

86.05

68.02

\section{Validation}

\begin{tabular}{lll} 
MolProbity Score & 2.61 & 2.69 \\
\hline Clashscore & 12.44 & 10.95 \\
\hline Poor rotamers (\%) & 5.49 & 6.70
\end{tabular}

\section{Ramachandran plot}

\begin{tabular}{lll} 
Favored (\%) & 93.14 & 91.42 \\
\hline Allowed (\%) & 6.75 & 8.39 \\
\hline Disallowed (\%) & 0.11 & 0.19
\end{tabular}

Despite nuclease treatment of the cell lysate, upon 3D reconstruction of the core structure, nucleic acid was clearly visible indicating that throughout the purification process the core enzyme remained tightly bound to nucleic acids which protected them from nuclease treatment. Therefore, the structure presented represents an elongation complex (EC) with non-specified nucleic acid sequence (i.e. the reconstructed density shows an average of random sequences). Typically, the subunit composition of core bacterial RNAP is represented as $a_{2} \beta \beta \bigotimes \omega$, but in previous work we identified an additional small subunit called $\varepsilon$ was present in $B$. subtilis RNAP in addition to $\omega^{19}$. However, holoenzyme preparations from the strain (LK637, $\Delta \delta$; Methods) used in this study lacked $\varepsilon$ (Extended Data Fig. 1a) and so the core structure is presented lacking this subunit (although $\varepsilon$ is present in the structure of RNAP core in complex with HelD, Extended Data Fig. 4a and b, see below). Previous studies have also shown deletion of $\varepsilon$ causes no detectable phenotype or change in gene expression profiles, and RNAP core preparations lacking $\varepsilon$ have indistinguishable activity compared to those that do contain it ${ }^{19,20}$. Comparison of EC and RNAP-HelD complexes showed no significant structural differences in the region where $\varepsilon$ binds and so in Fig. $1 \mathrm{~b}$ the $\varepsilon$ binding site is indicated as a dotted circle, and in Extended Data Fig. $4 \mathrm{c} \varepsilon$ is shown as it is clear that RNAP isolated from $B$. subtilis is a heterogeneous mixture of core $\left(a_{2} \beta \beta^{\prime} \omega\right) \pm \delta, \varepsilon$, and HelD, in addition to multiple different $\sigma$ factors ${ }^{21}$.

The EC is $150 \AA \times 112 \AA \times 123 \AA(L \times W \times H$, Table 1$)$, and is broadly comparable to the dimensions of core/Elongation complexes from other species $(157 \times 153 \times 136 \AA$; E. coli 6 ALF, $183 \times 107 \times 115 \AA$; 
Mycobacterium smegmatis 6F6W, and $170.1 \times 110.1 \times 127.8 \AA$; Thermus thermophilus 205I) ${ }^{22-24}$, although it appears to be more slender and elongated than the roughly globular $E$. coli, and shorter than the $M$. smegmatis and $T$. thermophilus enzymes due to the lack of insertion sequences.

Due to the high level of sequence conservation amongst RNAPs, the overall structure of the EC was similar to those from other organisms and largely consistent with homology models used in previous work on structure/function studies with $B$. subtilis RNAP ${ }^{25-28}$. However, modelling had been unable to establish the structure of the $\sim 180$ amino acid $\beta \ln 5$ insertion in the $\beta 2$ lobe. The $\beta 2$ lobe is one of the least well-conserved regions of bacterial RNAPs, and is a hot-spot for the presence of lineage-specific insertions ${ }^{17}$ (Extended Data Fig. 4c). The only other region that was significantly different to other bacterial RNAPs was a 10 amino acid loop from $\beta$ E696-G705 that protrudes from the bottom of the enzyme (Fig. 1C, Extended Data Fig. 4c). Refinement and building sequence into the resulting density indicated the $B$. subtilis $\beta 2$ lobe is a continuous globular structure and that the $\beta \ln 5$ insertion increases the size asymmetry between the $\beta$ lobes compared to other Gram positive RNAPs such as those from $M$. smegmatis and M. tuberculosis ${ }^{23,29}$ (Fig. 1b, Extended Data Fig. 4c). Searches using DALI ${ }^{30}$ found no structural matches to the $\beta \ln 5$ insertion leaving its function similarly enigmatic to those of most other lineage-specific insertions.

The absence of lineage-specific insertions perhaps helps to account for the additional subunits found in the Firmicutes such as $\delta$ and $\varepsilon$, and this and the accompanying paper by Pei et al. identify the location of these subunits. This suggestion is potentially supported by examination of the $T$. thermophilus structure around its $\beta \ln 10$ and $\beta \ln 12$ insertions that localise to a region very close to the $\varepsilon$ binding site. Superimposition of $\varepsilon$ into the $T$. thermophilus EC structure shows steric clashes between $\varepsilon$ and the insertions (Extended Data Fig. 4d) raising the possibility that they serve similar functions. The location of $\varepsilon$ also corresponds to that of a domain of archaeal and eukaryotic Rpo3/RPB3 subunits associated with enzyme stability (insert, Extended Data Fig. 4c) and it is interesting to note that both B. subtilis (able to grow up to $\sim 52^{\circ} \mathrm{C}$ ) and the thermophile $T$. thermophilus (up to $\sim 79{ }^{\circ} \mathrm{C}$ ) have structural elements/subunits located in this area that links the $\alpha_{2}, \beta$, and $\beta^{\prime}$ subunits whereas the mesophilic $E$. coli and $M$. smegmatis do not.

The $\omega$ subunit in $B$. subtilis is 67 amino acids $v s$ the 80 amino acid length of $E$. coli $\omega$. The main structural difference appears to be in the lack of a C-terminal a-helix which is prominent in $E$. coli RNAP, but lacking in B. subtilis and Mycobacterial structures. As with all other RNAP core and EC structures solved to date, the $\mathrm{C}$-terminal domains of the a subunits are not visible due to the flexible linker connecting the $\mathrm{N}$ - and $\mathrm{C}$-terminal domains.

Detailed examination of the elongation complex also revealed important features associated with mechanistic aspects of RNA synthesis. The density for fork-loop 2 (FL2) is well defined, consistent with its role in DNA strand separation on the downstream edge of the transcription bubble. The EC active site is very similar in structure to that reported previously for the T. thermophilus and E. coli ECs ${ }^{22,24}$ (205I, and 6ALF, respectively) and is in a post-translocation conformation with the $3^{\prime}$ end of the RNA transcript 
adjacent to the +1 site, with an unbent bridge-helix $(\mathrm{BH})$ and the trigger-loop $(T L)$ in the open conformation (Fig. 1d). This conformation is consistent with an elongation complex primed to receive an incoming NTP via the secondary channel.

FL2 residue $\beta$ R498 interacts with the ribose and phosphate moieties of the final base in the non-template DNA strand prior to strand separation and formation of the transcription bubble and likely acts to facilitate formation of the downstream edge of the transcription bubble (Fig. 1d). The template base in the +1 site is held in position for base-pairing with the incoming substrate NTP through interaction with the highly conserved T794 and $\mathrm{A795}$ of the $\mathrm{BH}$, and may also be stabilised through stacking with the base in the -1 position (Fig. 1d). $\beta$ R496 of FL2 interacts with the phosphodiester backbone of RNA bases 4 and 5 of the new transcript (Fig. 1d). In addition, residues Q469, P520, E521, N524, I528, K924 and $\mathrm{K} 932$ of the rifampicin binding pocket of the $\beta$ subunit form numerous interactions with the newly formed transcript (RNA residues $1-5$ ) as has been previously reported ${ }^{31,32}$. The salt bridge between $\beta$ R800 and $\beta^{\prime}$ D245 that closes the primary channel off from the RNA exit channel ${ }^{33,34}$ is clearly visible confirming that the elements on the upstream side of the transcription bubble, the rudder and lid, that are responsible for facilitating reannealing of the template and non-template strands and guiding RNA into the exit channel are in positions consistent with these assigned roles (Fig. 1e).

Electron density for RNA beyond the 8th nucleotide is poor, preventing further mapping of the transcript up to and through the exit channel. Likewise, density for DNA on the upstream side is poorly defined consistent with conformational flexibility in this region of RNAP ${ }^{26}$. Structural modelling, and comparison with ECs from other organisms, ${ }^{22,24}$ is consistent with there being sufficient space for a transcription bubble comprising a 9 bp template DNA-RNA hybrid prior to upstream DNA strand re-annealment and entry of the transcript into the exit channel guided by hydrophobic interaction with conserved $\beta^{\prime}$ lid residues V242 and L244 (dotted box, Fig. 1e). The 9th RNA-DNA base pair has likely been degraded by nuclease activity during preparation of the complex. Overall, this structure serves as a valuable resource for structure-function studies with RNAP from the Firmicutes as well as being a reference structure to enable full understanding of the conformational changes involved in transcription complex recycling induced upon binding to HelD (below).

\section{The structure of an RNAP-HelD transcription recycling complex}

RNAP-HelD complexes were isolated from a culture of $B$. subtilis carrying a deletion of the rpoE gene that encodes the $\delta$ subunit, shown previously to act synergistically with HelD ${ }^{12}$ (Extended Data Fig. 1). HelD itself is required for transcription complex recycling, and can perform this function independently of $\delta^{12}$ which is absent in many organisms that contain genes encoding HelD proteins (e.g. Clostridia). The purified complex stimulated transcription six-fold relative to purified RNAP core in multi-round transcription assays, $\sim 2-3$ times greater than is observed with in vitro assembled complexes ${ }^{12}$ (see above), establishing its biological activity (Fig. 2a). 
We determined the structure of the RNAP-HelD complex using single particle analysis cryo-electron microscopy (cryo-EM) to 3.36 Å resolution (Extended Data Fig. 3), followed by atomic modelling (Fig. 2b, Table 1; Extended Data Movie 2). Strikingly, the structure showed that HelD, which is located on the downstream side of RNAP, has two arm domains that penetrate deep into the primary and secondary channels of RNAP (clamp arm; CA, and secondary channel arm; SCA, respectively, Fig. 2b-d), which account for the strong HelD-RNAP interaction ${ }^{12,13,35}$. The native RNAP-HelD preparation also contained the RNAP $\varepsilon$ subunit ${ }^{19}$ and showed it bound on the downstream side of RNAP in a concave space between the two $a, \beta$, and $\beta^{\prime}$ subunits (Fig. 2b; see Extended Data Fig. 4).

HelD itself has an unusual 4-domain structure (Fig. 3a and b). The first 203 amino acids (aa) form the secondary channel arm (SCA), which is joined to a super-family 1 (SF1) 1A domain (aa 204-291 and 539-610). In SF1 helicases, domain $1 \mathrm{~A}$ is split by the insertion of a 1B domain associated with helicase function ${ }^{36}$, but in HelD it is split by the clamp arm (CA; aa 292-538). Residues 610-774 form a continuous SF1 2A domain, which is usually split by a 2B insertion in SF1 helicases, that represents the 'head' of HelD. The overall appearance of the protein is that of a torso and head (domains 1A and 2A, respectively) flanked by a pair of muscular arms (SCA and CA), giving it a rather thuggish appearance (Fig. 3b and c).

Remarkably, although HelD is widely distributed amongst Gram-positive bacteria, the distinctive arm domains represent the regions of lowest sequence conservation despite being responsible for the majority of interactions with RNAP as well as for its transcription recycling activity ${ }^{13}$ (Fig. 3a, Extended Data Figs. 5 and 6, Supplementary Information Fig. 1). It is also clear that there are at least two distinct classes of HelD (Classes I and II, Extended data Figs. 5, 6, Table 1); Class I is represented by the B. subtilis protein and is present in the low $\mathrm{G}+\mathrm{C}$ Gram positives, whereas Class II is represented by the $M$. smegmatis protein (see accompanying paper by Kouba et al.), and is present in the high $\mathrm{G}+\mathrm{C}$ Grampositives. Some organisms contain multiple copies of HelD (e.g. Lactobacillus plantarum, Class I; Nonomuraea wenchangensis, Class II; Extended Data Fig. 5), and even within the same organism, sequence conservation between the copies is relatively low in the SCA and CA domains (Supplementary Information Fig. 2). These observations suggest that the function of the arm domains is centred around mechanical work rather than the formation of highly-conserved functionally-significant interprotein interactions.

Despite the clear separation into two classes, sequence alignment allowed the identification of conserved motifs common to all HelD proteins (Fig. 3a; Extended Data Table 1). The transcription recycling function of HelD is dependent on ATP hydrolysis ${ }^{12}$, and ATP-binding motifs are located in the $1 \mathrm{~A}$ (torso) domain (cyan residues, Fig. $3 a$ and $b$ ). The remaining conserved motifs form a network of interactions that are mainly centred in the region between the SCA and 1A domains, with the absolutely conserved residue W137 in a hydrophobic pocket between them (purple residues, Fig. 3a and c). These extensive interactions anchor the SCA to the 1A domain, helping to couple ATP hydrolysis to mechanical movement of the CA (see below). 


\section{HelD causes major conformational changes in RNAP}

Comparison of the core elements of the EC and RNAP-HelD structures $\left(a_{2} \beta \beta^{\prime} \omega\right.$ subunits) shows HelD causes a dramatic conformational change mainly due to the opening of the $\beta^{\prime}$ clamp by the CA, with very little change elsewhere (Fig. 4a and b; Extended Data Movie 3, and see below). PISA ${ }^{37}$ was used to analyse protein-protein contacts in the RNAP-HelD and elongation complexes (Supplementary Information Table 1). Complexation with HelD reduces the contact area between RNAP subunits $\beta$ and $\beta^{\prime}$ by over $6 \%$ while other contact areas remain similar, consistent with the extensive conformational change caused to the EC upon binding of HelD.

As part of transcription complex recycling, the elongating RNA as well as the DNA template needs to dissociate from RNAP. RNA passes through the exit channel on the upstream side of RNAP. There was no major conformational change to elements at the entry of the exit channel other than those that are translocated as part of the opening of the $\beta^{\prime}$ clamp (Fig. 4). The translocation of the $\beta^{\prime}$ clamp results in breaking of the conserved salt bridge between $\beta$ R800 and $\beta^{\prime}$ D245 that is important in guiding RNA into the exit channel ${ }^{33,34}$, increasing the width of the aperture from 11 to $20 \AA\left(a_{c}-a_{c}\right.$; Fig. 4c, Extended Data Movie 3$)$. This separation, along with widening of the primary channel, facilitates RNA exit from the complex.

The most dramatic effect of HelD on RNAP is the widening of the primary channel from 21 to $47 \AA$ between $\beta 2$ lobe P242 and $\beta^{\prime}$ clamp helix N283, that would cause a loss of contact with DNA in the primary channel, enabling recycling of RNAP (Fig. $4 \mathrm{~b}$ and c). This is facilitated by the proximity of the CA to the SW5 region of the $\beta^{\prime}$ clamp, that acts as a hinge during clamp movement ${ }^{18,38}$ (Extended Data Fig. 7, Movie 3).

Detailed examination of the SCA and CA interactions with RNAP enabled us to define the molecular events that occur during transcription complex recycling. The Gre factors and DksA are known to bind in the secondary channel of RNAP via a pair of anti-parallel a helices ${ }^{39,40}$. The tip of these helices reside close to, but on the downstream side of the catalytic $\mathrm{Mg}^{2+}$. The SCA of HelD bears superficial similarity to GreB/DskA, but is longer and the tip extends past the catalytic $\mathrm{Mg}^{2+}$ (Fig. 5a). The acidic tip (D56 and D57) will electrostatically repel the transcript upon penetration of the SCA into the active site, with the SCA causing significant steric clashes with the transcript and template DNA strand when fully inserted (Fig. 5a). The bridge-helix and trigger-loop, are dynamic structures that play a key role in the transcription cycle ${ }^{41}$; the entry of the SCA into the secondary channel causes partial folding of the open trigger-loop conformation observed in the EC structure and a major distortion of the bridge-helix that would sterically clash with the template DNA in the active site (Fig. 5a; Extended Data Movie 3). Thus, the SCA tip itself, in combination with the distortion its insertion causes in the bridge-helix, will result in physical displacement of template DNA and RNA from the active site of RNAP, facilitated by electrostatic repulsion between the acidic SCA tip residues and the transcript. 
The fully inserted tip of the SCA is in close proximity to the absolutely conserved active-site $\beta_{447}^{\prime} N A D F D G D{ }_{453}$, forming a network of interactions around this motif, but does not directly interact with either the catalytic $\mathrm{Mg}^{2+}$ or the Asp residues that coordinate it (Fig. 5b, Supplementary Information Table 2). Thus, upon dissociation of HelD, the core RNAP would be competent for re-use in transcription, as seen in the transcription recycling assays in Figs. 1a and 2a. Finally, insertion of the SCA into the secondary channel would block NTP entry into the active site.

The salt-bridge and $\mathrm{H}$-bond contacts the CA makes with the $\beta^{\prime}$ clamp are listed in Supplementary Information Table 2, but the bulk of interactions are made by hydrophobic residues with little sequence conservation between even closely-related genera (Supplementary Information Fig. 1 and Table 3, Extended Data Fig. 6a). This region is the location of an insertion that spans across the primary channel towards the active site in Class II HelD proteins (see accompanying paper by Kouba et al.; Extended Data Fig. 6b). The tip of this insertion has a similar location to the tip of the SCA of B. subtilis Class I HelD and is also acidic, suggesting electrostatic repulsion of nucleic acids is also important in the activity of Class II HelDs. In our Class I HelD structure, the site of this insertion is close to an area of density in the cryo-EM reconstruction that at low threshold values could be consistent with the presence of nucleic acid (Extended Data Fig. 8). Examination of the surface charge of HelD revealed a region of high overall positive charge on the inside of the CA. Superposition with the nucleic acids from the EC show that this positively-charged patch is in a position where it could interact with the downstream dsDNA (Extended Data Fig. 8a), consistent with nucleic acid-binding data ${ }^{13}$. It is also possible that this patch may be important for interaction with the unstructured negatively-charged C-terminal domain of $\delta$ which acts synergistically with HelD during transcription complex recycling ${ }^{12}$ (see accompanying paper by Pei et al.). The end of the CA forms a relatively flat $\sim 320 \AA^{2}$ surface that acts as a platform to push up against the $\beta^{\prime}$ clamp, resulting in loss of contact with the DNA bound in the EC (Fig. 2b-d, Fig. 4, Extended Data Movie $3)$. Therefore, the purpose of the CA appears to involve the opening of the $\beta^{\prime}$ clamp through brute force rather than by the formation of a highly-specific network of conserved interactions.

\section{Movement of the clamp arm of HelD drives conformational change in RNAP}

Closer examination of the RNAP-HelD complex using 3D variability analysis (3DVA) ${ }^{42}$ allowed identification of regions of conformational flexibility that underpin the dynamic processes of HelD activity in transcription recycling. Overall, the region behind SW5 towards the a dimer, including the secondary channel and SCA of HelD, showed little or no

conformational variability, but the primary channel encompassing elements of the $\beta 1$ and 2 lobes and the $\beta^{\prime}$ clamp did (Fig. 6a, Extended Data Movie 4).

The 3D variability analysis indicates that HelD causes the $\beta^{\prime}$ clamp to open and twist so that the downstream side of RNAP opens slightly (curved cyan arrow, Fig. 6a). At the same time, the $\beta 2$ lobe moves up (straight cyan arrow, Fig. 6a) along with a slight twisting of the $\beta 1$ lobe and $\beta$ flap (Fig. 6a, 
Extended Data Movie 4). With respect to HelD, there was no change in the SCA tip adjacent to the RNAP active site, but there was lateral movement of the portion located outside the secondary channel towards the $\beta^{\prime}$ jaw (Fig. 6b). This resulted in little, if any, conformational change in the hydrophobic 'cage' surrounding the conserved W137 residue. Accordingly, there was relatively little change in the torso (1A) domain and ATP-binding site, but the head (2A) domain moved away from the downstream side of RNAP (curved and straight orange arrows Fig. $6 a$ and b, respectively). The CA of HelD rises up and out slightly, causing the upward twist on the downstream side of the $\beta^{\prime}$ clamp (orange arrow, Fig. 6a; Extended Data Movie 4). Therefore, the results of the 3D variability analysis are consistent with the SCA acting as a wedge that permits conformational change through movement of the CA. The CA is located in a position equivalent to an SF1 helicase 1B domain that utilises ATP hydrolysis to undergo conformational changes required for helicase/translocase activity ${ }^{43,44}$ and ATP binding/hydrolysis is required for release of HelD (see accompanying papers by Kouba et al. and Pei et al.), most likely due to movement of the CA arm, consistent with the observed conformational flexibility in this region.

In our structure, and those of the accompanying papers by Pei et al. and Kouba et al., no density for any NTP, could be detected in the ATP binding site, even on addition of ATP or non-hydrolysable analogues. In order to bind ATP, domains 1A (torso) and 2A (head) need to rotationally open as observed for SF1 helicases ${ }^{45}$. Our 3DVA suggests this is most likely via movement of the 2A (head) domain (Fig. 6a and b). However, the sequence from F183-G190 linking the SCA to the 1A (torso) domain sterically blocks access to the ATP binding site and this 'gate' region will also need to open to allow ATP binding and subsequent ADP release (Extended Data Fig. 9). There is no intramolecular bonding between residues T185-1189 and either the SCA or IA (torso) domain, and this may provide the necessary flexibility for gate opening and closing. Given that the ATP binding site was not accessible in all of the structures that are forcing open the DNA binding clamp of RNAP, gate opening may be an event that occurs on conformational change of the $C A$ during nucleic acid release and RNAP recycling.

\section{Discussion}

These results provide the foundations for a model of HelD catalysed transcription recycling (Fig. 6c). HelD is present at low intracellular levels compared to RNAP ${ }^{12,46}$ and this may help restrict it to targeting ECs that have entered a long-term pause ${ }^{11}$. The SCA penetrates the secondary channel (Punch), displacing the transcript and template DNA, blocking the catalytic $\mathrm{Mg}^{2+}$ and NTP entry. Through conserved inter-domain interactions with the torso, the SCA anchors HelD on RNAP. HelD then forces the primary channel open through interaction of the CA with the $\beta^{\prime}$ clamp (Uppercut). The action of both arms serves to displace nucleic acids from the active site, through separation of the $\beta$-flap and $\beta^{\prime}$-clamp by $10 \AA$, and opening the primary channel by $\sim 36 \AA$. Dissociation of HelD follows conformational change and ATP binding/hydrolysis (see accompanying papers by Kouba et al. and Pei et al.) consistent with SF1 helicase dynamics that are transmitted to the $\mathrm{CA}^{43,44}$, closing of the primary channel, and recycling of RNAP. 


\section{Methods}

\section{Strains}

E. coli BL21 (DE3) was used for overproduction of core $B$. subtilis RNAP, HelD and $\sigma^{\mathrm{A}}$. RNAP holoenzyme $(\mathrm{HE})$ and HelD complexes were purified from $B$. subtilis LK637 ${ }^{47}$ carrying a deletion to the rpoE gene encoding $\delta$, and a $3^{\prime}$ his tag on the rpoC gene to facilitate purification.

\section{Plasmids}

Recombinant $B$. subtilis RNAP core $\left(\mathrm{a}_{2} \beta \beta \omega\right)$ has previously been overproduced using a two plasmid system ${ }^{20}$ although the use of two different plasmids could result in low yields if one plasmid was present at lower levels than the other during overproduction. To make the process more efficient a new single plasmid system was constructed. pNG $219^{20}$ containing $r p o A, r p o B$, and $r p o C$ was linearised with Notl, purified and used in a Gibson assembly reaction with a 356 bp gBlock $($ IDT, Singapore) construct comprising 40 bp $5^{\prime}$ and $3^{\prime}$ homology to the linearised pNG219 DNA flanking an additional phage T7 promoter, $\mathrm{Ncol}$ and $\mathrm{Xbal}$ restriction sites, a ribosome binding site and the $r p o Z$ gene. The resulting plasmid was named pNG1256 (sequence and plasmid DNA available through Addgene, ID 149710).

Plasmid template for the MGA transcription assay ${ }^{48}$ consists of a construct containing three strong consensus promoters, Thermus VV1-2/D2 ${ }^{49}$, pGP31 from Bacillus phage SP01 and LacUV5 that directed transcription of an array containing 12 direct repeats of the MGA sequence (5'ggatcccgactggcgagagccaggtaacgaatggatcctaaaaac-3') followed by an E. colitRNA-trp terminator. This construct was synthesised by GenScript and cloned into the EcoRV site of pUC57-Simple to give pNG1299, (sequence and plasmid available through Addgene, ID 149709). pNG1299 was propagated in E. coli NEB ${ }^{\circledR}$ Stable $(\mathrm{C} 3040 \mathrm{H}$, New England Biolabs Inc) to avoid recombination of the MGA array. Supercoiled plasmid template was prepared as described by ${ }^{50}$ using the reagents from an ISOLATE II Plasmid Mini Kit (BIO-52057, Bioline).

\section{B. subtilis core RNAP purification.}

A seed culture $(40 \mathrm{ml})$ of $E$. coli BL21(DE3) transformed with pNG1256 was grown in LB supplemented with $100 \mu \mathrm{g} / \mathrm{ml}$ ampicillin at $37^{\circ} \mathrm{C}$ to an $A_{600}$ of 0.5 and was used to inoculate $4 \mathrm{~L}$ of auto-induction medium ${ }^{51}$ supplemented with $100 \mu \mathrm{g} / \mathrm{ml}$ ampicillin. The culture was grown at $30^{\circ} \mathrm{C}$ for $30 \mathrm{~h}$, cells harvested by centrifugation at $4,000 \times \mathrm{g}, 4^{\circ} \mathrm{C}, 20 \mathrm{~min}$, and washed pellets stored at $-80^{\circ} \mathrm{C}$. The frozen cell pellet was resuspended in $100 \mathrm{ml}$ HisA buffer $\left(20 \mathrm{mM} \mathrm{KPO}_{4}\right.$ pH7.8, $500 \mathrm{mM} \mathrm{NaCl}, 20 \mathrm{mM}$ imidazole) supplemented with EDTA-free protease inhibitor cocktail ( $1 \mathrm{x}$ concentration S8830, Sigma-Aldrich) and $100 \mu \mathrm{l}$ of $4 \mathrm{mg} / \mathrm{ml}$ DNasel (DN25, Sigma-Aldrich) at $4{ }^{\circ} \mathrm{C}$. Cells were lysed by repeated passage through an Avestin C5 homogeniser at $\sim 20 \mathrm{kPa}$, and the lysate clarified by centrifugation at $16,000 \times \mathrm{g}, 4^{\circ} \mathrm{C}$, 20 min. 
The resulting supernatant was loaded onto a $5 \mathrm{ml}$ HisTrap FF column pre-equilibrated with HisA buffer. The column was washed with $4 \% \mathrm{HisB}$ (HisA $+500 \mathrm{mM}$ imidazole), and core RNAP eluted with $50 \% \mathrm{HisB}$. Following dialysis in TrisA (20 mM Tris- $\mathrm{HCl}$ pH 7.8, $150 \mathrm{mM} \mathrm{NaCl}, 10 \mathrm{mM} \mathrm{MgCl}_{2}, 1 \mathrm{mM}$ DTT) the sample was loaded onto a $1 \mathrm{ml}$ MonoQ column pre-equilibrated in TrisA. A gradient of $0-50 \%$ TrisA supplemented with $1 \mathrm{M} \mathrm{NaCl}$ over $10 \mathrm{ml}$ was used to elute proteins with RNAP core eluting as a peak at $0.35 \mathrm{M} \mathrm{NaCl}$.

Purified RNAP core was dialysed into cryo buffer $(20 \mathrm{mM}$ Tris- $\mathrm{HCl} \mathrm{pH} \mathrm{7.8,} 150 \mathrm{mM} \mathrm{NaCl}, 10 \mathrm{mM} \mathrm{MgCl}$, 1 mM DTT), concentrated with an Amicon ${ }^{\circledR}$ Ultra-15 Centrifugal Filter with a 3 KDa NMWCO (UFC900324, Merck Millipore) and small aliquots snap-frozen in $\mathrm{N}_{2}(\mathrm{I})$ and stored at $-80^{\circ} \mathrm{C}$. Two different concentrations of purified core were prepared at $23.3 \mathrm{mg} / \mathrm{ml}$ and $8.2 \mathrm{mg} / \mathrm{ml}$ for storage.

\section{Bacillus subtilis $\sigma^{A}$ purification}

$\sigma^{\mathrm{A}}$ was overproduced and purified as described previously by ${ }^{25}$ with the following modifications; after HisTrap FF column purification, fractions containing $\sigma^{A}$ were dialysed overnight into $50 \mathrm{mM} \mathrm{NaH}_{2} \mathrm{PO}_{4} \mathrm{pH}$ 8.0, $150 \mathrm{mM} \mathrm{NaCl}$. Dialysate was loaded onto a Mono Q 5/50 GL column (17516601, GE Life Sciences) at a rate $0.5 \mathrm{ml} / \mathrm{min}$, and washed with $50 \mathrm{mM} \mathrm{NaH}_{2} \mathrm{PO}_{4} \mathrm{pH} 8.0,150 \mathrm{mM} \mathrm{NaCl}$ for $10 \mathrm{~mL}$ at a flow rate of

$0.5 \mathrm{ml} / \mathrm{min}$. $\sigma^{A}$ was eluted with a gradient of $150-500 \mathrm{mM} \mathrm{NaCl}$ over $20 \mathrm{~mL}$ followed by a step to $1 \mathrm{M}$ $\mathrm{NaCl}$ for $3 \mathrm{ml}$. Fractions containing $\sigma^{\mathrm{A}}$ were dialysed into $50 \mathrm{mM} \mathrm{NaH}_{2} \mathrm{PO}_{4} \mathrm{pH} 8.0,150 \mathrm{mM} \mathrm{NaCl}$ and concentrated with an Amicon ${ }^{\circledR}$ Ultra-15 Centrifugal Filter with a 3 kDa NMWCO. Concentrated samples were snap-frozen in $\mathrm{N}_{2}(\mathrm{I})$ and stored at $-80{ }^{\circ} \mathrm{C}$ before use.

\section{B. subtilis HelD Purification}

HelD was overproduced and purified as described by previously ${ }^{12}$ with the following modifications; after HisTrap FF column purification fractions containing HelD were dialysed overnight at $4{ }^{\circ} \mathrm{C}$ into $(20 \mathrm{mM}$ Tris-HCl pH7.8, $10 \mathrm{mM} \mathrm{MgCl}_{2}, 150 \mathrm{mM} \mathrm{NaCl}, 5 \mathrm{mM}$ DTT) and applied to a $1 \mathrm{ml} \mathrm{HiTrap} \mathrm{Heparin} \mathrm{HP}$ column (17040601, GE Lifesciences) at a flow rate of $1 \mathrm{ml} / \mathrm{min}$. The column was washed with $5 \mathrm{ml}$ $20 \mathrm{mM}$ Tris- $\mathrm{HCl} \mathrm{pH7.8,} 10 \mathrm{mM} \mathrm{MgCl}, 150 \mathrm{mM} \mathrm{NaCl}$ ) at $1 \mathrm{ml} / \mathrm{min}$ and eluted with a gradient of $150 \mathrm{mM}-$ $1000 \mathrm{mM} \mathrm{NaCl}$ over $20 \mathrm{ml}$. Fractions containing HelD were dialysed overnight into Dialysis Buffer and concentrated using an Amicon® Ultra-15 Centrifugal Filter with a 3 kDa NMWCO (UFC900324, Merck Millipore). The concentrated sample was snap-frozen in $\mathrm{N}_{2}(\mathrm{I})$.

\section{B. subtilis HelD-RNAP complex}

B. subtilis $\mathrm{LK} 637^{47}(\Delta \delta)$ was grown at $45^{\circ} \mathrm{C}$ in LB in baffled flasks to maximise aeration to late exponential phase, cells pelleted by centrifugation, and washed pellets stored at $-80^{\circ} \mathrm{C}$. Frozen pellets 
from $8 \mathrm{~L}$ culture were resuspended in $50 \mathrm{ml}$ HisA buffer (above) supplemented with EDTA-free protease inhibitor cocktail ( $1.8 \times$ concentration S8830, Sigma-Aldrich) at $4{ }^{\circ} \mathrm{C}$, and lysed by multiple passage through an Avestin C5 homogeniser at $\sim 25 \mathrm{kPa}$, and the lysate clarified by centrifugation at $16,000 \times \mathrm{g}$, $4^{\circ} \mathrm{C}, 20 \mathrm{~min}$.

RNAP was purified using a $5 \mathrm{ml}$ HisTrap FF as above and the eluted sample dialysed overnight against QA buffer (20 mM Tris-HCl pH 7.8, $150 \mathrm{mM}, 10 \mathrm{mM} \mathrm{MgCl} 2,1 \mathrm{mM}$ EDTA, $5 \mathrm{mM}$ DTT) at $4{ }^{\circ} \mathrm{C}$. Following clarification by centrifugation, the overnight dialysate was loaded onto a $1 \mathrm{ml}$ MonoQ column preequilibrated in QA buffer. RNAP was eluted using a $13.5 \mathrm{ml} 150-500 \mathrm{mM} \mathrm{NaCl}$ gradient in QA buffer. RNAP containing fractions were pooled and dialysed overnight at $4{ }^{\circ} \mathrm{C}$ against QA buffer prior to loading onto a $1 \mathrm{ml} \mathrm{HiTrap} \mathrm{Heparin} \mathrm{HP} \mathrm{column} \mathrm{pre-equilibrated} \mathrm{in} \mathrm{QA} \mathrm{buffer.} \mathrm{RNAP} \mathrm{was} \mathrm{eluted} \mathrm{as} \mathrm{two} \mathrm{peaks} \mathrm{using}$ a 150-1000 mM NaCl gradient over $15 \mathrm{ml}$. The first peak corresponded to Holoenzyme ( $650 \mathrm{mM} \mathrm{NaCl})$, and the second, minor peak, to the HelD complex ( $950 \mathrm{mM} \mathrm{NaCl})$.

Holoenzyme and HelD complex fractions were dialysed separately against buffer QA overnight at $4{ }^{\circ} \mathrm{C}$, concentrated and small aliquots snap-frozen in $\mathrm{N}_{2}(\mathrm{l})$ and stored at $-80^{\circ} \mathrm{C}$. Holoenzyme fractions were $7.46 \mathrm{mg} / \mathrm{ml}$ and HelD complex fractions $2.57 \mathrm{mg} / \mathrm{ml}$.

\section{Transcription assays}

An in vitro transcription assay adapted from ${ }^{48}$ was used to assay the activities of RNAP and RNAP-HelD complexes. Briefly, $20 \mu \mathrm{L}$ transcription reactions containing $80 \mathrm{nM}$ of core RNAP, $240 \mathrm{nM}$ of $\sigma^{\mathrm{A}}$ and 0 $2560 \mathrm{nM}$ of HelD in transcription buffer (40 mM Tris- $\mathrm{HCl} \mathrm{pH} \mathrm{7.5,} 50 \mathrm{mM} \mathrm{KCl}, 10 \mathrm{mM} \mathrm{MgCl}_{2} 0.02 \%$ (v/v) Triton-X100, 8 mM DTT) were assembled in a well of a black, half area, flat-bottomed, non-binding 96 well microplate (CLS3686, Corning) and were incubated for $15 \mathrm{~min}$ at $37^{\circ} \mathrm{C}$ with shaking. To initiate the transcription reaction 20 $\mu \mathrm{L}$ of start solution (1 mM rNTPs (N0466S, New England Biolabs Inc), $10 \mathrm{nM}$ pNG1299, in transcription buffer) was added. The plate was sealed with a clear adhesive plate seal (WHA-7704-0001, Whatman) and incubated with shaking at $37^{\circ} \mathrm{C}$ for $15 \mathrm{~min}$. Reactions were stopped by the addition of $40 \mu \mathrm{L}$ of ice-cold stop solution (10 mM Tris-HCl pH8, 1 mM EDTA, $144 \mu \mathrm{M}$ malachite green oxalate salt (M6880, Sigma-Aldrich)) and developed on ice for $5 \mathrm{~min}$. The plate was read using a Pherastar FS (BMG Labtech) using a $610 \mathrm{~nm}$ excitation, $675 \mathrm{~nm}$ emission optical module. Percentage transcription was calculated relative to a 1:0 RNAP:HelD reaction. Each experiment was performed three times in technical duplicate.

\section{Preparation of Cryo-EM grids and cryo-electron microscopy}

Between 2 and $2.5 \mu \mathrm{l}$ of $2.57 \mathrm{mg} / \mathrm{ml}$ RNAP-HelD complex or $3.0 \mathrm{mg} / \mathrm{ml}$ EC diluted in QA buffer were deposited onto glow-discharged UltraFoil 1.2/1.3 or Quantifoil 1.2/1.3 cryo-electron microscopy grids and blotted for 5 seconds before plunge freezing into liquid ethane using a Mark IV Vitrobot (FEI). Data were collected on a Titan Krios (Thermo Fisher) electron microscope operated at $300 \mathrm{kV}$ and equipped with a Gatan BioQuantum LS 967 energy filter and Gatan K2 Summit detector, operated in unfiltered mode. Data were collected in electron counting mode at a pixel size of $0.84 \AA$ /pixel and a calibrated sample-to-pixel 
magnification of $59524 \mathrm{x}$, (microscope user interface listed magnification, $165000 \times$ EFTEM). For the HelD complex, movies were collected as a series of 60 frames with a total accumulated dose of $63.6 \mathrm{e}^{-} /$ $\AA^{2}$. For the RNA polymerase elongation complex, movies were collected as a series of 40 frames and a total accumulated dose of $52.2 \mathrm{e}^{-} / \AA^{2}$. For both datasets, data were collected using automated data collection in EPU, with a defocus range of $0.6-2.8 \mu \mathrm{m}$. Approximately $60 \%$ of the data for both the RNAP HelD complex and the RNAP elongation complex were collected at $20^{\circ}$ stage tilt to compensate for the effects of preferred particle orientation.

\section{Image processing of the HelD complex}

A total of 4331 images (1859 at $0^{\circ}$ tilt, 2472 at $20^{\circ}$ tilt) were collected for the RNAP-HelD complex. All image processing was performed in RELION $3.1^{52}$ unless otherwise indicated. Movies were aligned and dose-weighted using MotionCor $2^{53}$ before contrast-transfer function (CTF) estimation was performed on the motion-corrected images using GCTF ${ }^{54}$. Particle picking was performed in $\mathrm{CrYOLO}^{55}$ using a pretrained general model which had been refined using a subset of manually picked training data. Particle coordinates were contrast-inverted, normalised and extracted in RELION. Following particle picking, a total of 580,468 particles were extracted and subjected to several rounds of 2D classification for initial cleaning of the particle data, which resulted in a subset of 379,179 particles. Subsequent rounds of 3D classification isolated a smaller subset of 65,356 particles. Due to moderate preferred orientation of the specimen, a large portion of the particles were excluded to minimise resolution anisotropy in the final reconstruction. These particles were then subjected to iterative Bayesian polishing and CTF refinement with higher-order aberration correction in RELION until further processing ceased to yield an increase in resolution. Following refinement of the per-particle motion and CTF parameters, post-processing of the final reconstruction yielded a resolution of $3.36 \AA$ as determined by the Gold-standard Fourier-Shell Correlation (GSFSC) criterion in RELION. The final refined map was then subjected to density modification and automated model-based based sharpening in Phenix ${ }^{56,57}$ (see Extended Data Fig. 2).

Following reconstruction in RELION, the final subset of 65,356 particles was subjected to 3D variability analysis in Cryosparc $^{42}$. 3D variability analysis was performed solving for 3 conformational modes, and the results visualised in ChimeraX ${ }^{58}$.

\section{Image processing of the Elongation complex}

A total of 5185 images ( 2955 at $0^{\circ}$ tilt, 2230 at $20^{\circ}$ tilt) were collected for the elongation complex. All processing was performed as described for the HelD complex above unless otherwise indicated. Following particle picking, a total of 1,069,336 particles were extracted in RELION and subjected to several rounds of 2D classification to obtain a smaller subset of 355,795 particles. This subset was subjected to several rounds of 3D classification to remove incomplete particles and over-represented orientations to isolate a final subset of 58,854 particles. These particles were then subjected to Bayesian polishing, CTF-refinement and postprocessing as for the HelD complex above. Following postprocessing, the final reconstruction yielded a resolution of $3.36 \AA$ as determined by the GSFSC criterion in RELION. As 
for the RNAP-HelD complex, the final refined map was then subjected to density modification and automated model-based based sharpening in Phenix ${ }^{57,59}$ (see Extended Data Fig. 3).

\section{Structure building and refinement}

Initial model building for the core RNA polymerase subunits commenced using a homology model generated previously ${ }^{19}$, which was fitted into the density of the HelD complex using rigid body fitting in CHIMERA followed by molecular-dynamics flexible fitting in NAMD ${ }^{60}$. The model was subject to cycles of manual model building in $\mathrm{COOT}^{61}$ followed by refinement by phenix.real_space_refine. De novo atomic modelling for the HelD subunit was performed by initial modelling of HelD in Phenix using phenix.map_to_model ${ }^{59}$, followed by manual refinement in COOT $^{61}$.

The epsilon subunit was modelled based on homology with a known structure from Geobacillus stearothermophilus (PDB ID: 4NJC). Refinement of the elongation complex commenced using the RNAPHelD model, which was placed into density using phenix.dock_in_map, followed by cycles of manual building in COOT and refinement in phenix.real_space_refine. Arbitary sequence was built for nucleic acids. All models were further refined in ISOLDE ${ }^{62}$ and phenix.real_space_refine until deemed final.

\section{Sequence analysis}

B. subtilis HelD sequence (UniProtKB - 032215) was used to identify similar proteins using the NCBI CDART search program ${ }^{63}$. Sequences from diverse organisms were selected from the 24426 hits defined as HelD-related helicases for alignment using ClustalX $2.1^{64}$. Sequences were also selected from the DUF4968 domain-containing protein (74 hits) and the multispecies: DUF4968 domain-containing protein (37 hits) categories for characterisation of their HelD-like sequences. Phylogenetic trees were constructed using NCBI COBALT ${ }^{65}$, and sequence conservation mapped to structure using ConSurf ${ }^{66}$.

\section{Reporting summary}

Further information is available in the Nature Research Reporting Summary linked to this paper.

\section{Data availability}

CryoEM maps were deposited in the Electron Microscopy Data Bank (https://www.ebi.ac.uk/pdbe/emdb/) under accession codes EMD-21921 (RNAP-HelD) and EMD-21920 (RNAP elongation complex), and will be released upon publication. Structure coordinates have been deposited in the RCSB Protein Data Bank (https://www.rcsb.org/) with accession codes 6WVK (RNAP-HelD) and 6WVJ (dimeric RNAP elongation complex), and will be released upon publication. Plasmids pNG1256 and pNG1299 are available from Addgene (https://www.addgene.org) under accession numbers 149710 and 149709, respectively and will be made available upon publication. All other data supporting the findings of this study are available from the corresponding author on request.

\section{Declarations}




\section{Acknowledgements}

We would like to thank Profs Nick Dixon and Rick Lewis for helpful comments on the manuscript, and members of our respective laboratories and those of Dr Libor Krasny and Prof Markus Wahl for discussions. P.J.L. acknowledges the assistance of Sarah Pichereau during the purification of RNAP-HelD complexes. This work was supported by grants from the Priority Research Centre for Drug Discovery, University of Newcastle (P.J.L), NUW Alliance (G1801287 to P.J.L, A.J.O and G.T.), and NHMRC (GNT1184012 to G.T.). T.N. and M.M. were funded through PhD scholarships from the Australian Government.

\section{Author information}

\section{Affiliations}

Molecular Microbiology Laboratory, School of Environmental and Life Sciences, University of Newcastle

Michael Miller

Catherine Dawson

Peter J. Lewis

Molecular Horizons and School of Chemistry and Molecular Bioscience, University of Wollongong, and Illawarra Health and Medical Research Institute, Wollongong

Timothy Newing

Aaron J. Oakley

Simon H.J. Brown

James C. Bouwer

Gökhan Tolun

\section{Contributions}

P.J. L, M.M. and C.D. cloned genes, produced proteins/complexes and performed experiments. T.N. prepared and imaged cryo-EM samples with S.H.J.B and J.B. T.N. processed cryo-EM data with S.H.J.B., P.J.L., and G.T., and built atomic models with A.J.O. and G.T. All authors contributed to the analysis of the data and the interpretation of the results. P.J.L. wrote the manuscript with contributions from the other authors. P.J.L. and G.T. supervised work in their respective groups. P.J.L. conceived and coordinated the project.

\section{Corresponding author}


Correspondence to Peter J. Lewis or Gökhan Tolun.

\section{Ethics declarations}

\section{Competing interests}

The authors declare no competing interests.

\section{References}

1. Pomerantz, R. T. \& O'Donnell, M. The replisome uses mRNA as a primer after colliding with RNA polymerase. Nature 456, 762-766, doi:10.1038/nature07527 (2008).

2. Pomerantz, R. T. \& O'Donnell, M. Direct restart of a replication fork stalled by a head-on RNA polymerase. Science 327, 590-592, doi:10.1126/science.1179595 (2010).

3. Gupta, M. K. et al. Protein-DNA complexes are the primary sources of replication fork pausing in Escherichia coli. Proc Natl Acad Sci U S A 110, 7252-7257, doi:10.1073/pnas.1303890110 (2013).

4. Rocha, E. P. C. The replication-related organization of bacterial genomes. Microbiology 150, 16091627, doi:10.1099/mic.0.26974-0 (2004).

5. Adelman, K. \& Lis, J. T. Promoter-proximal pausing of RNA polymerase II: emerging roles in metazoans. Nat Rev Genet 13, 720-731, doi:10.1038/nrg3293 2012.

6. Epshtein, V.et al. UvrD facilitates DNA repair by pulling RNA polymerase backwards. Nature $\mathbf{5 0 5}, \mathbf{3 7 2 -}$ 377, doi:10.1038/nature12928 2014.

7. Ghodke, H., Ho, H. N. \& van Oijen, A. M. Single-molecule live-cell imaging visualizes parallel pathways of prokaryotic nucleotide excision repair. Nat Commun 11, 1477, doi:10.1038/s41467-020-15179-y (2020).

8. Ho, H. N., van Oijen, A. M. \& Ghodke, H. The transcription-repair coupling factor Mfd associates with RNA polymerase in the absence of exogenous damage. Nat Commun 9, 1570, doi:10.1038/s41467-01803790-z (2018).

9. Sanders, K.et al. The structure and function of an RNA polymerase interaction domain in the PcrA/UvrD helicase. Nucleic Acids Res 45, 3875-3887, doi:10.1093/nar/gkx074 (2017).

10. Sikova, M.et al. The torpedo effect in Bacillus subtilis: RNase J1 resolves stalled transcription complexes. EMBO J 39, e102500, doi:10.15252/embj.2019102500 (2020).

11. Saba, J.et al. The elemental mechanism of transcriptional pausing. Elife 8, doi:10.7554/eLife.40981 (2019). 
12. Wiedermannova, J.et al. Characterization of HelD, an interacting partner of RNA polymerase from Bacillus subtilis. Nucleic Acids Res 42, 5151-5163, doi:10.1093/nar/gku113 (2014).

13. Koval, T.et al. Domain structure of HelD, an interaction partner of Bacillus subtilis RNA polymerase. FEBS Lett 593, 996-1005, doi:10.1002/1873-3468.13385 (2019).

14. Hawkins, M.et al. Direct removal of RNA polymerase barriers to replication by accessory replicative helicases. Nucleic Acids Res 47, 5100-5113, doi:10.1093/nar/gkz170 (2019).

15. Liu, B., Zuo, Y. \& Steitz, T. A. Structural basis for transcription reactivation by RapA. Proc Natl Acad Sci U S A 112, 2006-2010, doi:10.1073/pnas.1417152112 (2015).

16. Xu, J.et al. Structural basis for the initiation of eukaryotic transcription-coupled DNA repair.Nature 551, 653-657, doi:10.1038/nature24658 (2017).

17. Lane, W. J. \& Darst, S. A. Molecular evolution of multisubunit RNA polymerases:sequence analysis. $J$ Mol Bio/ 395, 671-685, doi:10.1016/j.jmb.2009.10.062

2010.

18. Lane, W. J. \& Darst, S. A. Molecular evolution of multisubunit RNA polymerases:structural analysis. J Mol Bio/ 395, 686-704, doi:10.1016/j.jmb.2009.10.063 (2010).

19. Keller, A. N.et al. epsilon, a new subunit of RNA polymerase found in gram-positive bacteria. $J$ Bacterio/ 196, 3622-3632, doi:10.1128/jb.02020-14 (2014).

20. Yang, X. \& Lewis, P. J. Overproduction and purification of recombinant Bacillus subtilis RNA polymerase. Protein Expr Purif 59, 86-93, doi:10.1016/j.pep.2008.01.006 (2008).

21. Helmann, J. D. Purification of Bacillus subtilis RNA polymerase and associated factors. Methods Enzymo/ 370, 10-24, doi:10.1016/S0076-6879(03)70002-0

2003.

22. Kang, J. Y.et al. Structural basis of transcription arrest by coliphage HK022 Nun in an Escherichia coli RNA polymerase elongation complex. Elife 6, doi:10.7554/eLife.25478 (2017).

23. Kouba, T.et al. The Core and Holoenzyme Forms of RNA Polymerase from Mycobacterium smegmatis. J Bacterio/ 201, doi:10.1128/JB.00583-18 (2019).

24. Vassylyev, D. G., Vassylyeva, M. N., Perederina, A., Tahirov, T. H. \& Artsimovitch,I. Structural basis for transcription elongation by bacterial RNA polymerase. Nature $448,157-162$, doi:10.1038/nature05932 (2007). 
25. Johnston, E. B., Lewis, P. J. \& Griffith, R. The interaction of Bacillus subtilis sigmaA with RNA polymerase. Protein Sci 18, 2287-2297, doi:10.1002/pro.239 (2009).

26. Ma, C.et al. RNA polymerase-induced remodelling of NusA produces a pause enhancement complex.Nucleic Acids Res 43, 2829-2840, doi:10.1093/nar/gkv108 (2015).

27. Ma, C.et al. Inhibitors of bacterial transcription initiation complex formation. ACS Chem Bio/ 8, 19721980, doi:10.1021/cb400231p (2013).

28. Yang, X.et al. The structure of bacterial RNA polymerase in complex with the essential transcription elongation factor NusA. EMBO Rep 10, 997-1002, doi:

10. 1038/embor.2009.155 (2009).

29. Lin, W.et al. Structural Basis of Mycobacterium tuberculosis Transcription and Transcription Inhibition.Mol Cell 66, 169-179 e168, doi:10.1016/j.molcel.2017.03.001 (2017).

30. Holm, L. Benchmarking fold detection by DaliLite v.5. Bioinformatics 35, 5326-5327, doi:10.1093/bioinformatics/btz536 (2019).

31. Artsimovitch, l.et al. Allosteric modulation of the RNA polymerase catalytic reaction is an essential component of transcription control by rifamycins. Cell 122, 351-363, doi:10.1016/j.cell.2005.07.014 (2005).

32. Molodtsov, V.et al. X-ray crystal structures of the Escherichia coli RNA polymerase in complex with benzoxazinorifamycins.J Med Chem 56, 4758-4763, doi:10.1021/jm4004889 (2013).

33. Naryshkina, T., Kuznedelov, K. \& Severinov, K. The role of the largest RNA polymerase subunit lid element in preventing the formation of extended RNA-DNA hybrid. J Mol Bio/ 361, 634-643, doi:10.1016/j.jmb.2006.05.034 (2006).

34. Toulokhonov, I. \& Landick, R. The role of the lid element in transcription by E. coli RNA polymerase. $J$ Mol Bio/ 361, 644-658, doi:10.1016/j.jmb.2006.06.071 (2006).

35. Delumeau, O.et al. The dynamic protein partnership of RNA polymerase in Bacillus subtilis.

Proteomics 11, 2992-3001, doi:10.1002/pmic.201000790 (2011).

36. Singleton, M. R., Dillingham, M. S. \& Wigley, D. B. Structure and mechanism of helicases and nucleic acid translocases. Annu Rev Biochem 76, 23-50, doi:10.1146/annurev.biochem.76.052305.115300 (2007).

37. Krissinel, E. \& Henrick, K. Inference of macromolecular assemblies from crystalline state. J Mol Biol 372, 774-797, doi:10.1016/j.jmb.2007.05.022 (2007). 
38. Gnatt, A. L., Cramer, P., Fu, J., Bushnell, D. A. \& Kornberg, R. D. Structural basis of transcription: an RNA polymerase II elongation complex at 3.3 A resolution.Science 292, 1876-1882, doi:10.1126/science.1059495 (2001).

39. Abdelkareem, M.et al. Structural Basis of Transcription: RNA Polymerase Backtracking and Its Reactivation.Mol Cell 75, 298-309 e294, doi:10.1016/j.molcel.2019.04.029 (2019).

40. Molodtsov, V.et al. Allosteric Effector ppGpp Potentiates the Inhibition of Transcript Initiation by DksA. Mol Cel/ 69, 828-839 e825, doi:10.1016/j.molcel.2018.01.035 (2018).

41. Brueckner, F., Ortiz, J. \& Cramer, P. A movie of the RNA polymerase nucleotide addition cycle. Curr Opin Struct Biol 19, 294-299, doi:10.1016/j.sbi.2009.04.005 (2009).

42. Punjani, A. \& Fleet, D. J. 3D Variability Analysis: Directly resolving continuous flexibility and discrete heterogeneity from single particle cryo-EM images. bioRxiv, 2020.2004.2008.032466, doi:10.1101/2020.04.08.032466 (2020).

43. Stelter, M., Acajjaoui, S., McSweeney, S. \& Timmins, J. Structural and mechanistic insight into DNA unwinding by Deinococcus radiodurans UvrD. PLoS One 8, e77364, doi:10.1371/journal.pone.0077364 (2013).

44. Velankar, S. S., Soultanas, P., Dillingham, M. S., Subramanya, H. S. \& Wigley,D. B. Crystal structures of complexes of PcrA DNA helicase with a DNA substrate indicate an inchworm mechanism. Cel/ 97, 75-84, doi:10.1016/s0092-8674(00)80716-3 (1999).

45. Yang, W. Lessons learned from UvrD helicase: mechanism for directional movement.Annu Rev Biophys 39, 367-385, doi:10.1146/annurev.biophys.093008.131415 (2010).

46. Nicolas, P.et al. Condition-dependent transcriptome reveals high-level regulatory architecture in Bacillus subtilis. Science 335, 1103-1106, doi:10.1126/science.1206848 (2012).

47. Rabatinova, A.et al. The delta subunit of RNA polymerase is required for rapid changes in gene expression and competitive fitness of the cell. J Bacterio/ 195, 2603-2611, doi:10.1128/JB.00188-13 (2013).

48. Scharf, N. T., Molodtsov, V., Kontos, A., Murakami, K. S. \& Garcia, G. A. Novel Chemical Scaffolds for Inhibition of Rifamycin-Resistant RNA Polymerase Discovered from High-Throughput Screening. SLAS Discov 22, 287-297, doi:10.1177/2472555216679994 (2017).

49. Peredultchuk, M., Vonstein, V. and Demirjian, D. C.. Thermus promoters for gene expression. USA patent (1999).

50. Pronobis, M. I., Deuitch, N. \& Peifer, M. The Miraprep: A Protocol that Uses a Miniprep Kit and Provides Maxiprep Yields. PLoS One 11, e0160509, doi:10.1371/journal.pone.0160509 (2016). 
51. Studier, F. W. Protein production by auto-induction in high density shaking cultures.Protein Expr Purif 41, 207-234 (2005).

52. Zivanov, J.et al. New tools for automated high-resolution cryo-EM structure determination in RELION3.Elife 7, doi:10.7554/eLife.42166 (2018).

53. Zheng, S. Q.et al. MotionCor2: anisotropic correction of beam-induced motion for improved cryoelectron microscopy. Nat Methods 14, 331-332, doi:10.1038/nmeth.4193 (2017).

54. Zhang, K. Gctf: Real-time CTF determination and correction. J Struct Biol 193, 1-12, doi:10.1016/j.jsb.2015.11.003 (2016).

55. Wagner, T.et al. SPHIRE-crYOLO is a fast and accurate fully automated particle picker for cryoEM.Commun Bio/2, 218, doi:10.1038/s42003-019-0437-z (2019).

56. Afonine, P. V.et al. New tools for the analysis and validation of cryo-EM maps and atomic models. Acta Crystallogr D Struct Bio/ 74, 814-840, doi:10.1107/S2059798318009324 (2018).

57. Terwilliger, T. C., Ludtke, S. J., Read, R. J., Adams, P. D. \& Afonine, P. V.Improvement of cryo-EM maps by density modification. bioRxiv, 845032, doi:10.1101/845032 (2020).

58. Goddard, T. D.et al. UCSF ChimeraX: Meeting modern challenges in visualization and analysis. Protein Sci 27, 14-25, doi:10.1002/pro.3235 (2018).

59. Terwilliger, T. C., Adams, P. D., Afonine, P. V. \& Sobolev, O. V. A fully automatic method yielding initial models from high-resolution cryo-electron microscopy maps. Nat Methods 15, 905-908, doi:10.1038/s41592-018-0173-1 (2018).

60. Trabuco, L. G., Villa, E., Mitra, K., Frank, J. \& Schulten, K. Flexible fitting of atomic structures into electron microscopy maps using molecular dynamics. Structure 16, 673-683, doi:10.1016/j.str.2008.03.005 (2008).

61. Emsley, P. \& Cowtan, K. Coot: model-building tools for molecular graphics. Acta Crystallogr D Biol Crystallogr 60, 2126-2132, doi:10.1107/S0907444904019158 (2004).

62. Croll, T. I. ISOLDE: a physically realistic environment for model building into low-resolution electrondensity maps. Acta Crystallogr D Struct Bio/ 74, 519-530, doi:10.1107/S2059798318002425 (2018).

63. Geer, L. Y., Domrachev, M., Lipman, D. J. \& Bryant, S. H. CDART: protein homology by domain architecture. Genome Res 12, 1619-1623, doi:10.1101/gr.278202 (2002).

64. Larkin, M. A.et al. Clustal W and Clustal X version 2.0. Bioinformatics 23, 2947-2948, doi:10.1093/bioinformatics/btm404 (2007). 
65. Papadopoulos, J. S. \& Agarwala, R. COBALT: constraint-based alignment tool for multiple protein sequences. Bioinformatics 23, 1073-1079, doi:10.1093/bioinformatics/btm076 (2007).

66. Ashkenazy, H.et al. ConSurf 2016: an improved methodology to estimate and visualize evolutionary conservation in macromolecules. Nucleic Acids Res 44, W344-350, doi:10.1093/nar/gkw408 (2016).

\section{Figures}




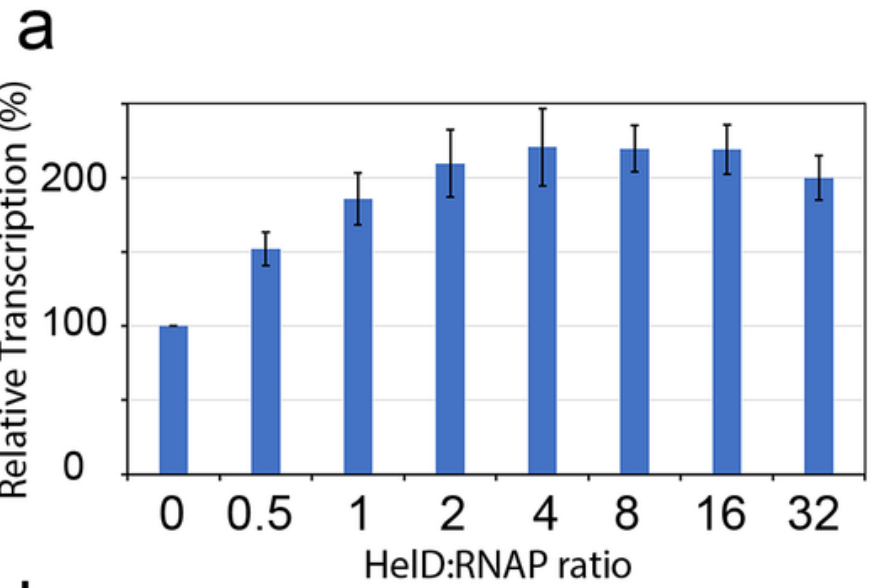

d

b

$3^{\prime}$
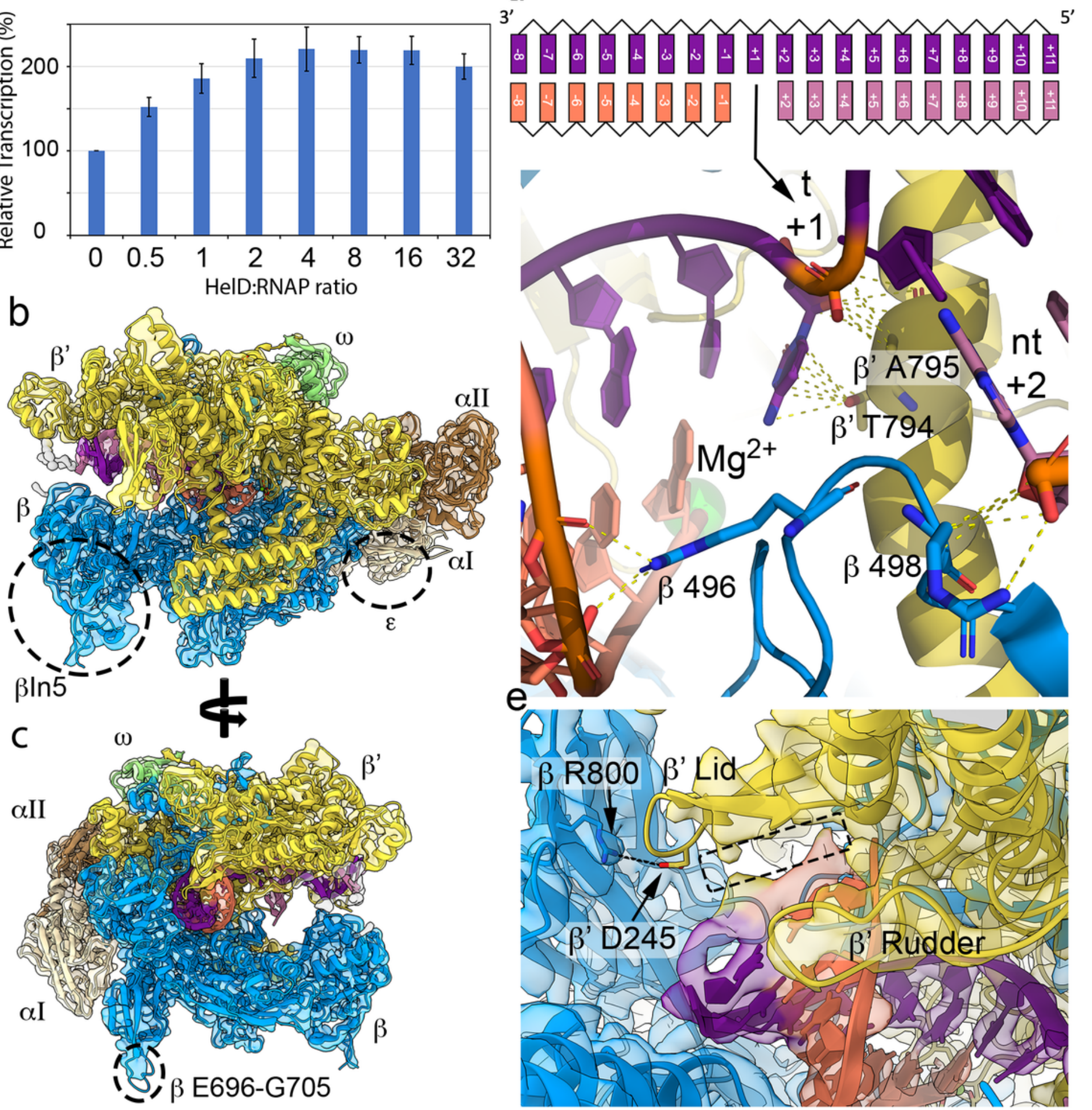

Figure 1

Structure of RNAP elongation complex. a, Multi-round transcription assays of purified RNAP core supplemented with increasing amounts of HelD (as a ratio with RNAP) showing HelD-dependent stimulation. Error bars, SD. Each experiment was performed three times in technical duplicate. b and c, Cryo-EM reconstruction of the RNAP elongation complex (EC). The electron density map is semitransparent in the same colours as the subunits in the cartoon structure: al beige, all brown, $\beta$ azure, $\beta^{\prime}$ 
yellow, $\omega$ light green, template DNA purple, non-template DNA pink, and RNA orange. The dotted circles show the location of the $\beta \ln 5$ insertion, $\varepsilon$ binding site (b) and $\beta$ E696-G705 insertion (c), respectively. $d$, Top shows a schematic of the nucleic acids coloured as in panels b and c. +1 represents the template DNA nucleotide positioned within the active site. + integers represent nucelotides base paried as DNA on the downstream side of RNAP, and -ve integers represent the DNA-RNA hybrid on the upstream side. The arrow indicates the position of the unpaired +1 nucleotide in the active site of RNAP in the lower part of the panel. Intermolecular bonds (polar and non-polar) are indicated by the dashed lines. Relevant template $(\mathrm{t})$ and non-template (nt) nucleotides are numbered appropriately and amino acids shown with the RNAP subunit as a prefix. The catalytic Mg2+ ion is shown as a green sphere to indicate the active site. e, An enlarged view of the upstream side of the EC close to the entry of the RNA exit channel. The salt bridge formed between $\beta$ R800 and $\beta^{\prime}$ D245 is shown as black dashed line. The space below the $\beta^{\prime}$ lid that could accommodate a 9th DNA-RNA base pair is shown as a dotted rectangle, with the $\beta^{\prime}$ rudder helping guide RNA towards the $\beta^{\prime}$ lid and the RNA exit channel.

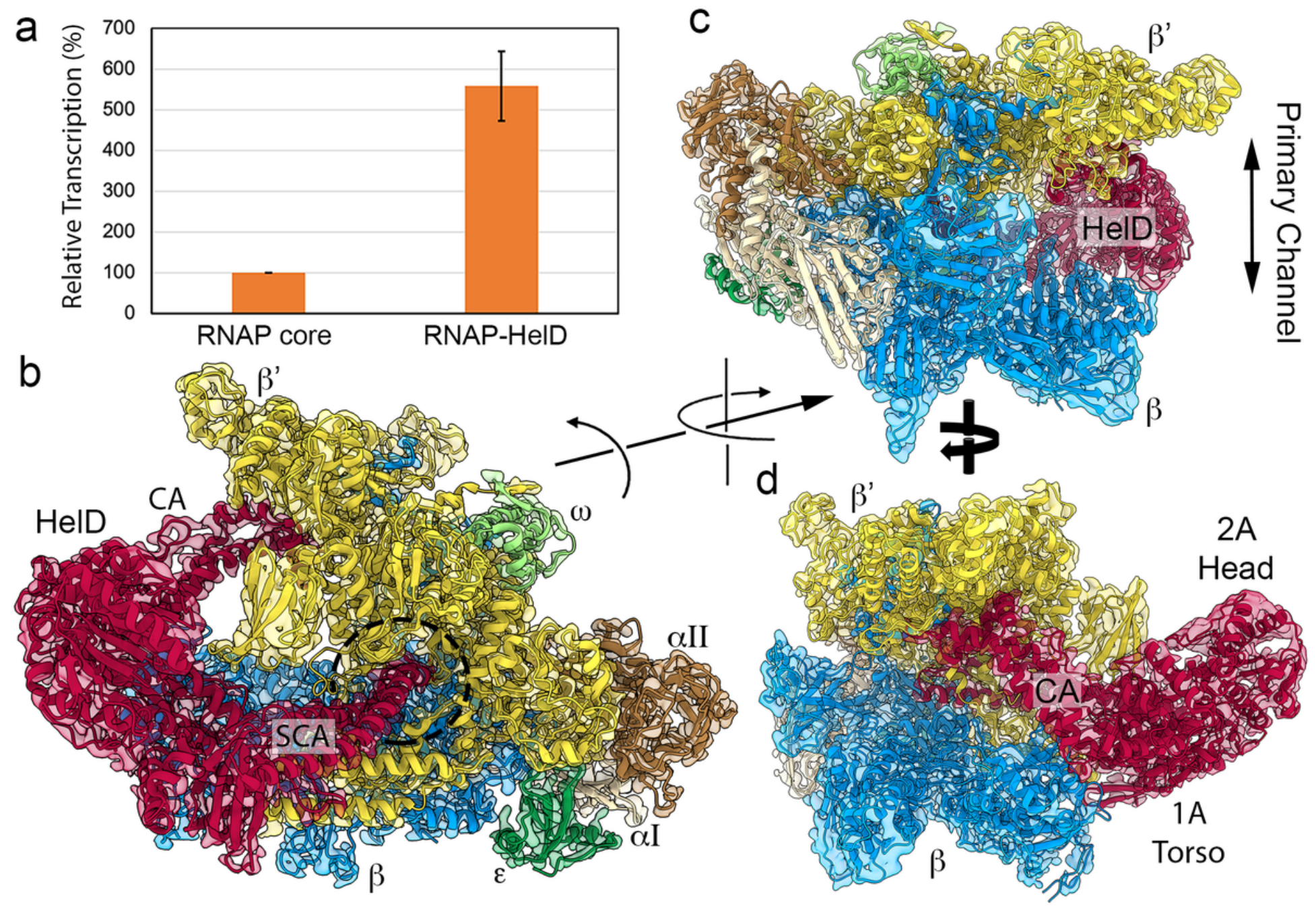

Figure 2

Structure of RNAP in complex with HelD. a, Results of a multi-round transcription assay (\% relative transcription) of purified RNAP core versus the RNAP-HelD complex purified from B. subtilis. Error bars, 
SD. Each experiment was performed three times in technical duplicate. b-d, Different views of the cryo-EM reconstruction of the RNAP-HelD complex. The electron density map is semi-transparent in the same colours as in Fig. 1 with the addition of $\varepsilon$ green, and HelD red. The clamp arm (CA), secondary channel arm (SCA), 1A Torso, and 2A Head domains are labelled. The primary channel that is formed between the $\beta$ and $\beta$ ' subunits is shown in $c$, and the secondary channel indicated by the dotted circle in $b$. The change in orientation between the views in panels $b$ and $c$ is indicated by the arrows, with the left side arrow indicating a rotation of the view in $b$ upwards from the $a 2 / \varepsilon$ end that would move the $\beta^{\prime}$ end down. The right arrow indicates the subsequent $165^{\circ}$ clockwise rotation of the complex to give the view in $\mathrm{c}$. The veiw in $\mathrm{d}$ is a simple $90^{\circ}$ rotation of the orientation in c.

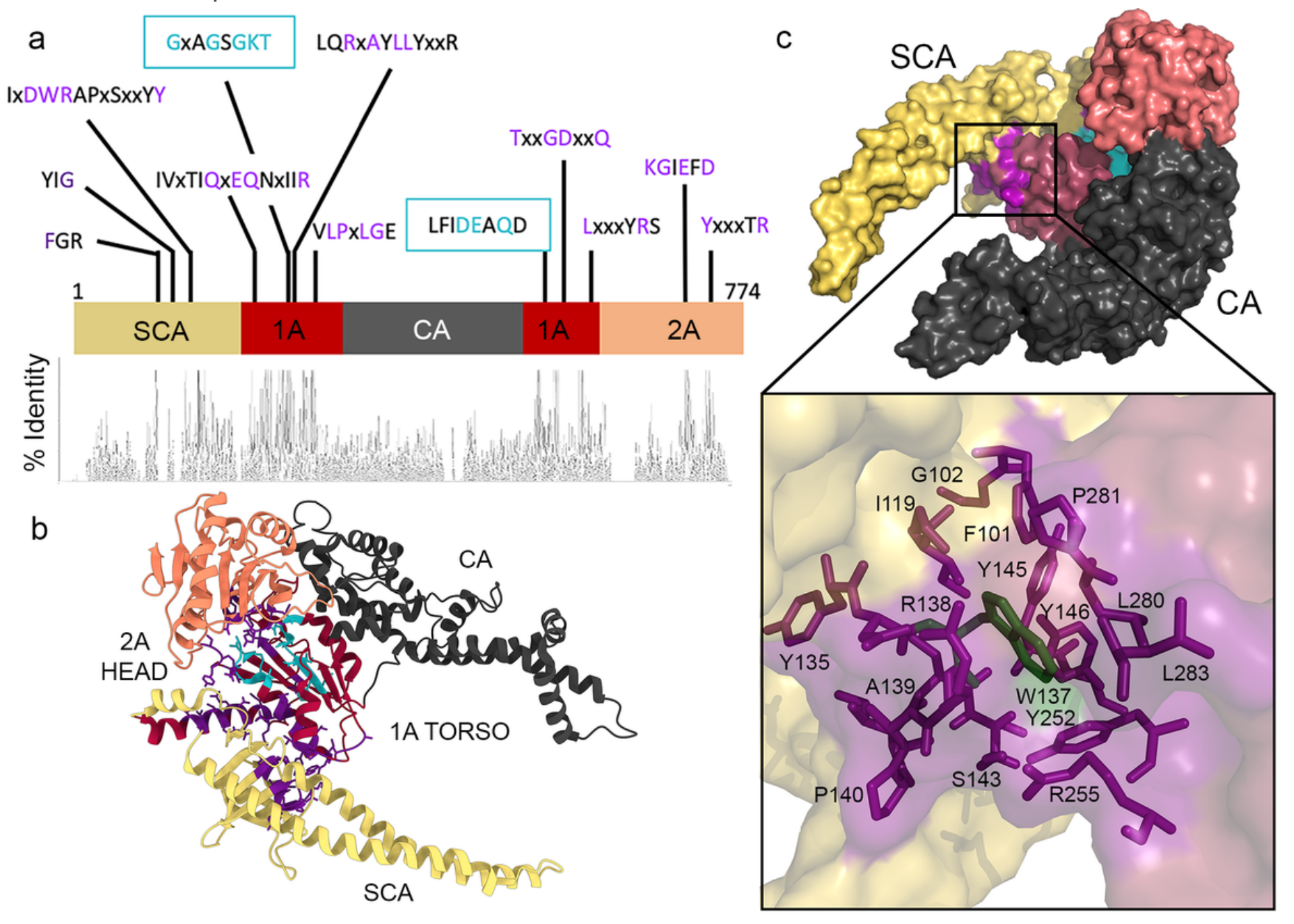

Figure 3

Fig 3.png 

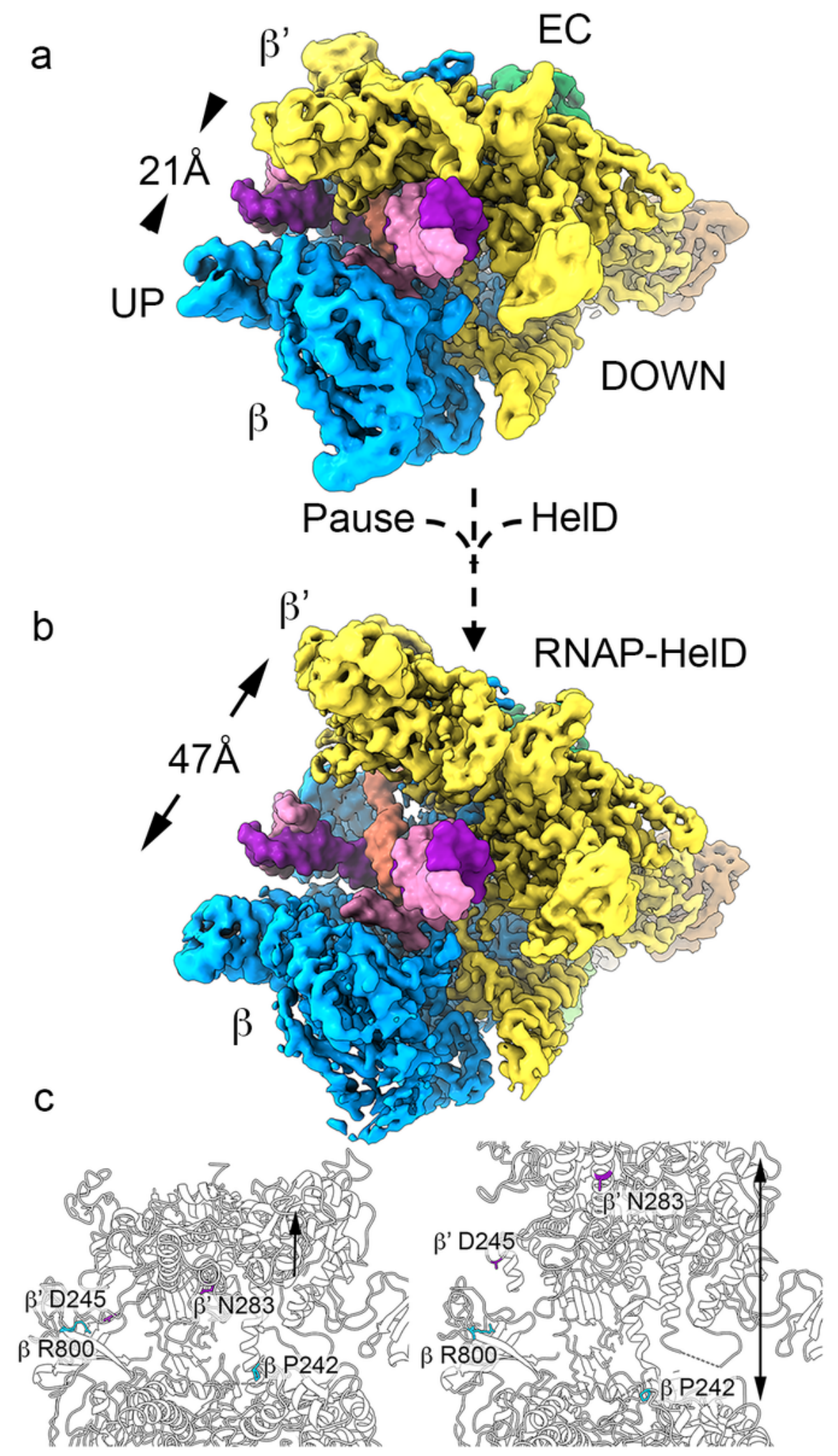

Figure 4

HelD induces a significant conformational change in RNAP. a and b, Cryo-EM electron density maps ( $a 2 \beta \beta \omega$ subunits only) of RNAP EC (a) and RNAP-HelD complex (b). HelD density has been removed for clarity, and nucleic acids from the E. coli EC (PDB ID 6ALF) superimposed over the B. subtilis EC nucleic acids as a visual aid for the scale of conformational changes that occur in the primary DNA-binding and RNA exit channels upon binding HelD. Nucleic acids are coloured the same as in Fig. 1. The longer RNA 
from the E. coli EC helps illustrate the increase in aperture of the RNA exit channel. $d$, left side EC, right side RNAP-HelD complex with HelD removed for clarity, with $\beta$ (cyan) and $\beta \bigotimes$ (purple) residues shown to illustrate the change in aperture of the RNA exit channel $\beta$ R800 and $\beta \triangle D 245$ and DNA binding channel $\beta$ $P 242$ and $\beta \square N 283$. Black arrow in left panel indicates the region of the $\beta \square$ clamp that is contacted by the HelD clamp arm and the double-ended arrow in the right panel indicates the movement of subunits away from each other on HelD binding.

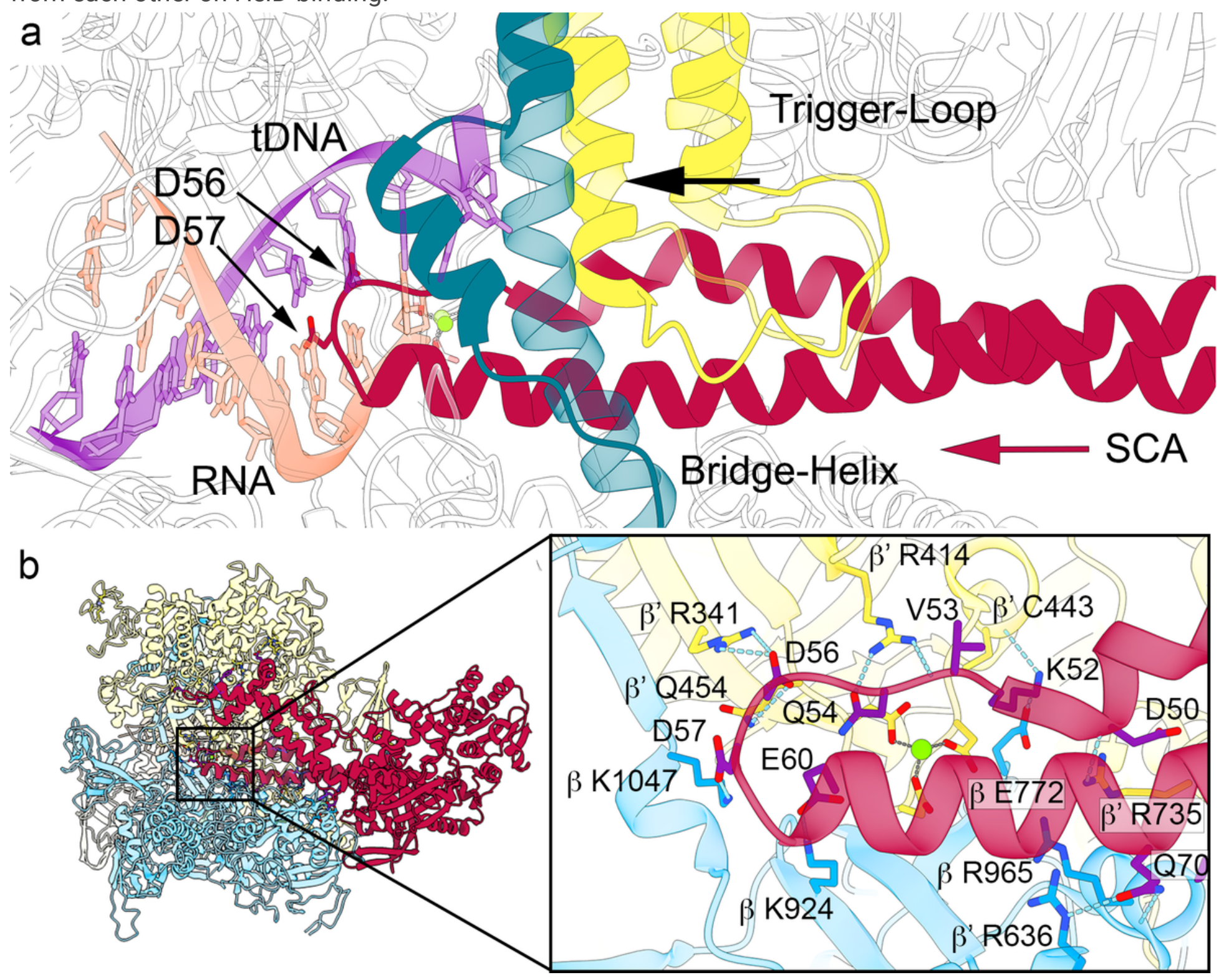

Figure 5

HelD interactions with RNAP. a, The SCA (red) following insertion (direction of red arrow) into the secondary channel. The acidic tip residues of the SCA, D56 and D57, are shown as sticks. EC elements are shown semi-transparent with nucleic acids coloured according to the scheme in Fig. 1. The bridgehelix (teal) and trigger-loop (yellow) elements that are distorted by the SCA are shown, with the direction of movement from EC to HelD complex indicated by the black arrow. The catalytic Mg2+ ion is shown as a green sphere. $b$, Hydrogen-bond and salt-bridge interactions (dashed blue lines) between the tip of the 
SCA (red semi-transparent cartoon with purple sticks) and RNAP. Subunit colouring is the same as in Fig. 1. The conserved active site Asp residues that chelate the catalytic Mg2+ (green sphere, grey dashed lines) are also shown to illustrate how the tip of the SCA cages but does not interact directly with residues involved in RNAP catalysis. The enlarged box on the right corresponds to the boxed region shown for the whole complex on the left.

a

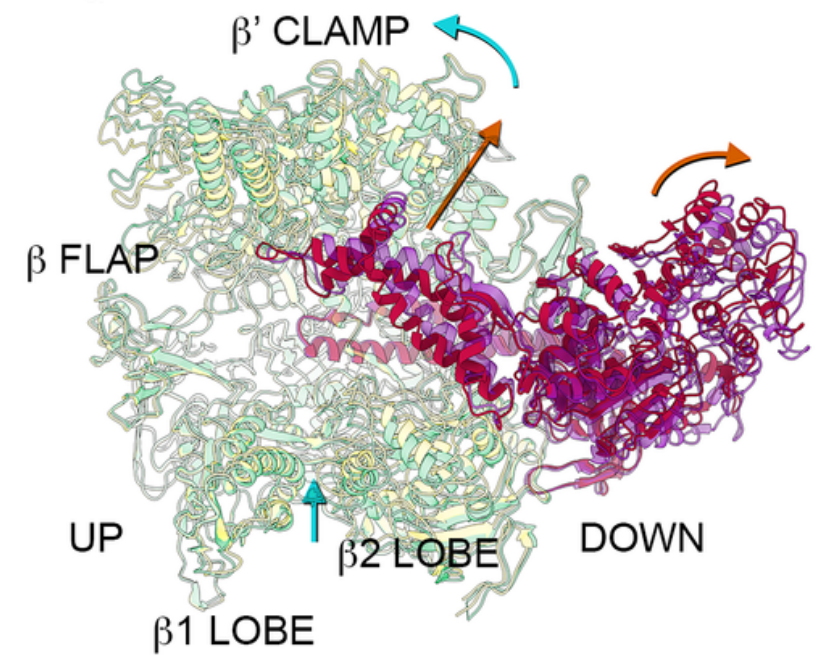

b
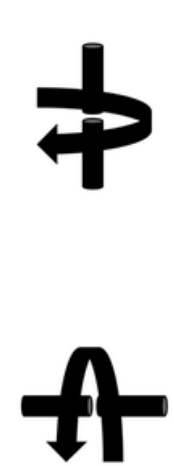

C
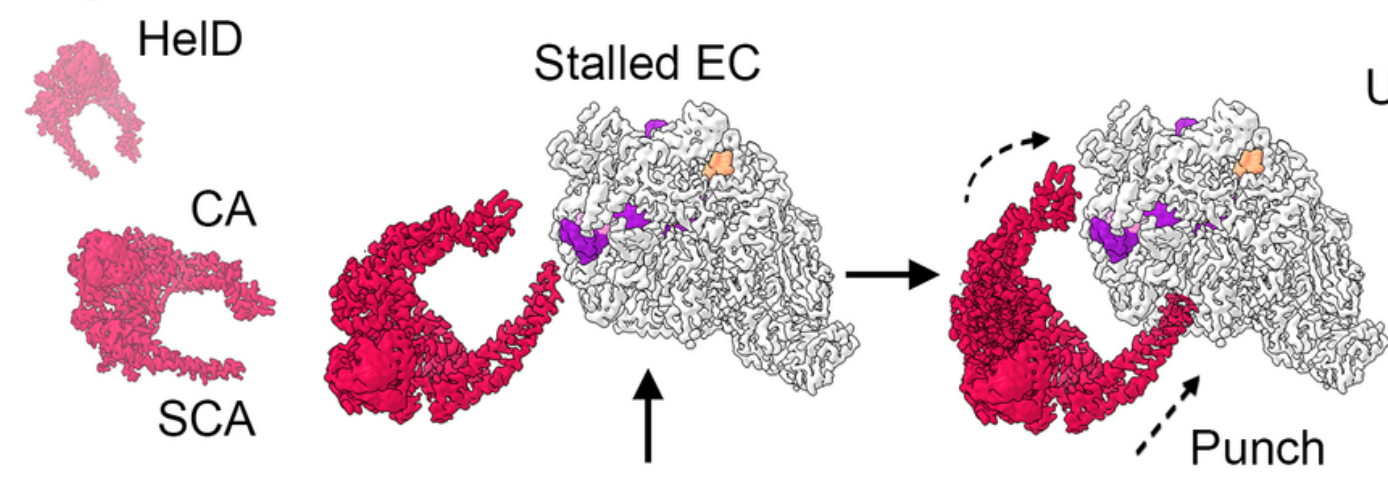

Uppercut
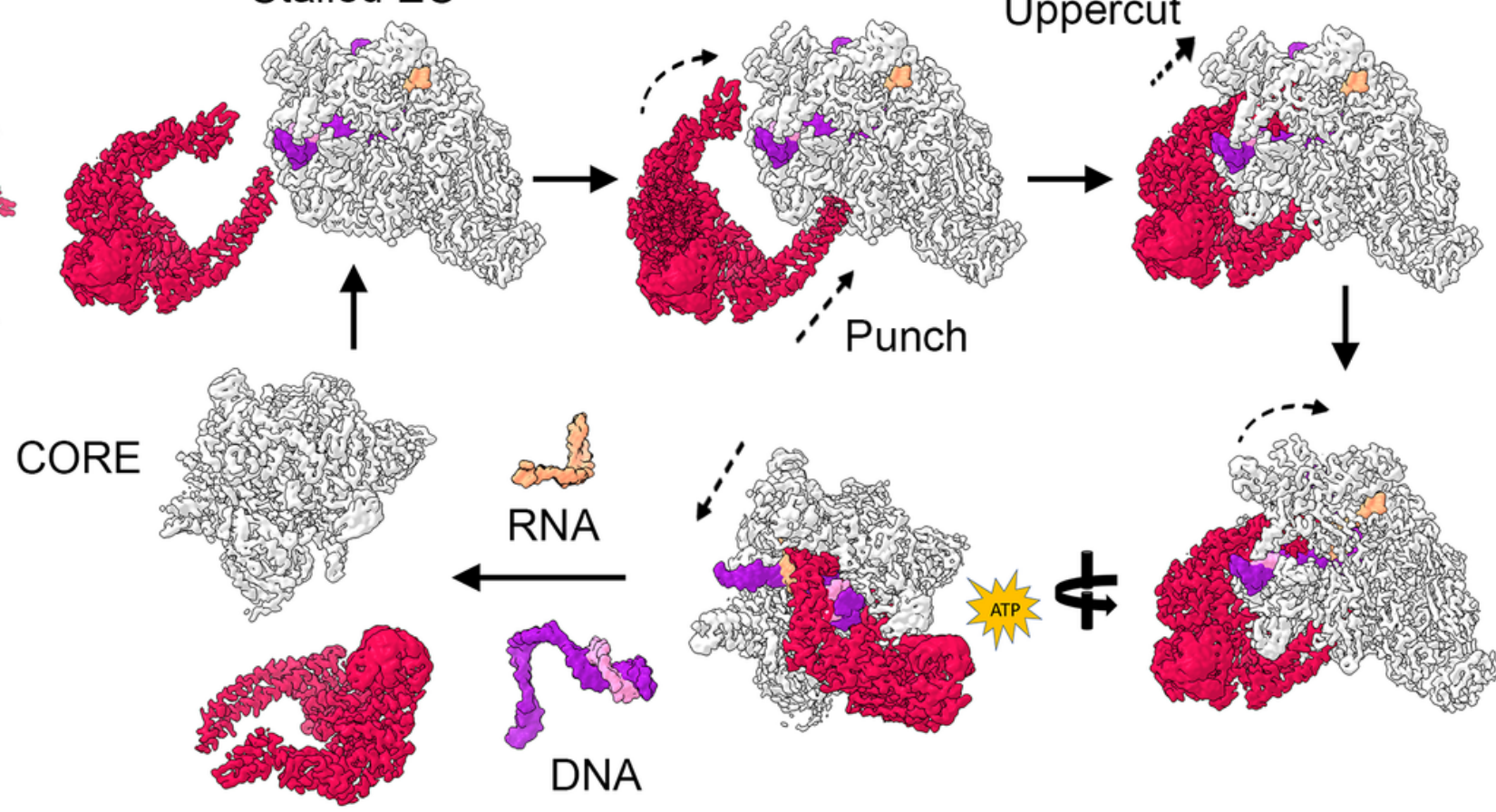

$\beta$ ' CLAMP

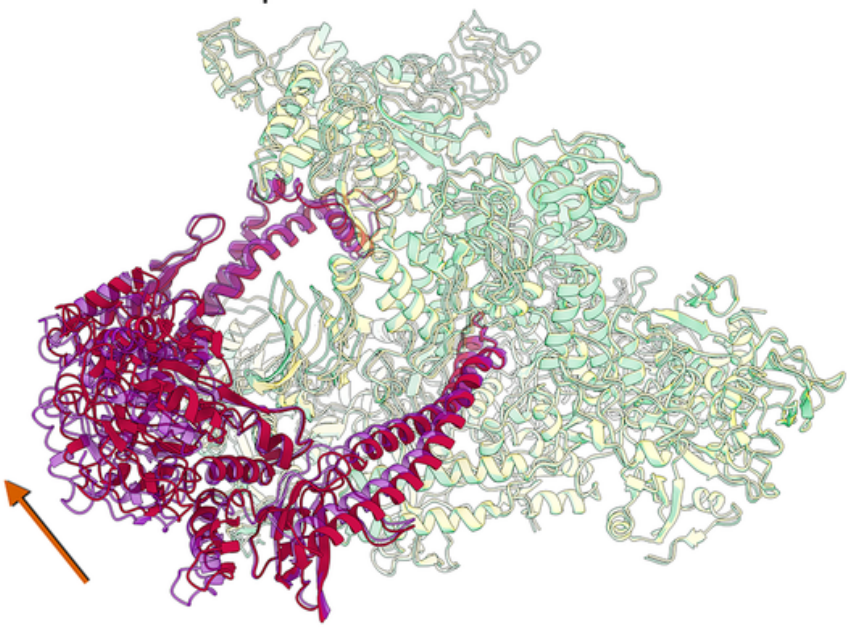


conformation of RNAP, and the purple HelD with the yellow RNAP. Orange arrows indicate the movement of the juxtaposed region of HelD, and cyan arrows regions of RNAP. Structural elements of RNAP referred to in the text are labelled as well as the up- (UP) and downstream (DOWN) sides of RNAP. c, Model for HelD-catalysed recycling. HelD is shown in red, RNAP in white, DNA in purple (template) and pink (nontemplate), and RNA in orange. Clockwise from top left; HelD locates a stalled EC and binds with the SCA penetrating the secondary channel and the CA moving into position on the $\beta^{\prime}$ clamp. The SCA is wedged deep within RNAP, and through conserved interactions with the $1 \mathrm{~A}$ torso domain, locks HelD in position. The contacts the CA makes with the $\beta^{\prime}$ clamp, open the DNA-binding and RNA-exit channels to enable dissociation of nucleic acids from the EC, possibly assisted by interaction of the DNA with the positivelycharged patch on the CA of HelD. HelD (and nucleic acid) dissociation is facilitated by the conformational changes facilitated by ATP binding/hydrolysis (ATP flash). Finally, core RNAP that has been released from the complex is free to enter a fresh round of transcription.

\section{Supplementary Files}

This is a list of supplementary files associated with this preprint. Click to download.

- Extendeddata.docx

- Supplementarylnfo.docx

- SupplementaryInformationFigure1.pdf

- SupplementarylnformationFigure2.pdf

- SupplementaryTable1.xlsx

- SupplementaryTable2.xlsx

- SupplementaryTable3.xlsx 